\title{
Die inklusive Gemeinde
}

\section{Eine Vision für gelingendes Leben vor Ort}

\section{ANDREAS STRUNK}

Prof. Dr. phil. Andreas Strunk, Dipl. Ing. ist Sozialpädagoge und Sozialplaner. Für die Gesellschaft für Innovation, Systementwicklung und Soziale Arbeit (GISAmbH) leitet er Projekte der Organisationsentwicklung und Sozialforschung. Weiterbildungen als Supervisor, lösungsorientierter Berater und Case Manager. Er ist Vorstandsmitglied im Deutschen Berufsverband für Soziale Arbeit (DBSH), Landesverband Baden-Württemberg.

E-Mail prof.strunk@t-online.de

\author{
Die Forderung nach »Inklusion« ist allgegenwärtig. \\ Das Recht zur Teilhabe wird am besten verwirklicht, \\ wenn vor Ort, in der Gemeinde, die erforderlichen \\ Bedingungen für die Zugehörigkeit von Menschen \\ zu Gesellschaft geschaffen werden.
}

Seit dem Jahre 2009 ist das »Übereinkommen der Vereinigten Nationen über die Rechte von Menschen mit Behinderungen (BRK) in Deutschland zum Bundesrecht geworden. Seitdem ist nicht nur in der Sozialwirtschaft dort vor allem in der Behindertenhilfe -, sondern auch im Bereich allgemeiner Sozialplanung ein hoher Innovationsdruck entstanden. Das kann u. a. an der Fachpolitischen Stellungnahme »Inklusive Sozialplanung " des Vereins für Sozialplanung e. V. deutlich werden. (1)

Der Grund liegt darin begründet, dass die UN-Behindertenrechtskonvention mit ihrem »Normalisierungsanspruch « generell Aussagen macht über die Entwicklung einer inklusiven Gemeinde. Im Artikel 1 wird der Zweck der Konvention beschrieben: "Zweck dieses Übereinkommens ist es, den vollen und gleichberechtigten Genuss aller Menschenrechte und Grundfreiheiten durch alle Menschen mit Behinderung zu fördern, zu schützen und zu gewährleisten und die Achtung der ihnen innewohnenden Würde zu fördern. Zu den Menschen mit Behinderung zählen Menschen, die langfristige körperliche, seelische, geistige oder Sinnesbeeinträchtigung haben, welche sie in Wechselwirkung mit verschiedenen Barrieren an der vollen, wirksamen und gleichberechtigten Teilhabe an der Gesellschaft hindern können.« (2)

In der UN-Behindertenrechtskonvention werden insgesamt 15 Arbeitsfelder aufgerufen; diese Arbeitsfelder sind:

• Hilfen im Vorschulalter, Frühförderung
- elementare Bildung und Erziehung, Kindertageseinrichtungen

- schulische Bildung und Erziehung

- allgemeine Bildung

- Zugänglichkeit

- persönliche Mobilität

- Gesundheit

- Arbeit und Beschäftigung

- Wohnen und Unterstützung im Alltag

- ältere und alte Menschen mit Behinderung

- Teilhabe am politischen und öffentlichen Leben

- Teilhabe am kulturellen Leben, sowie Erholung, Freizeit und Sport

- Habilitation und Rehabilitation

- Planung und Steuerung von Hilfen für Menschen mit Behinderung

- Monitoring und Evaluation

Als Beispiel für die Bedeutung der Gemeindeentwicklung kann der Artikel 19 benannt werden - Unabhängige Lebensführung und Einbeziehung in die Gemeinschaft:

"Die Vertragsstaaten diese Übereinkommens anerkennen das gleiche Recht aller Menschen mit Behinderung mit gleichen Wahlmöglichkeiten wie andere Menschen in der Gemeinschaft zu leben und treffen wirksame und geeignete Maßnahmen, um Menschen mit Behinderung den vollen Genuss dieses Rechtes und ihre volle Einbeziehung in die Gemeinschaft und Teilhabe an der Gemeinschaft zu erleichtern, indem sie unter anderem gewährleisten, dass

a) Menschen mit Behinderung gleichberechtigt die Möglichkeit haben, 
ihren Aufenthaltsort zu wählen und zu entscheiden wo und mit wem sie leben, und nicht verpflichtet sind, in besonderen Wohnformen zu leben;

b) Menschen mit Behinderungen $\mathrm{Zu}$ gang zu einer Reihe von gemeindenahen Unterstützungsdiensten zu Hause und in Einrichtungen sowie zu sonstigen gemeindenahen Unterstützungsdiensten haben, einschließlich der persönlichen Assistenz, die zur Unterstützung des Lebens in der Gemeinschaft und der Einbeziehung in die Gemeinschaft sowie zur Verhinderung von Isolation und Absonderung von der Gemeinschaft notwendig ist;

c) Gemeindenahe Dienstleistungen und Einrichtungen für die Allgemeinheit Menschen mit Behinderungen auf der Grundlage der Gleichberechtigung zur Verfügung stehen und ihren Bedürfnissen Rechnung tragen.« seling « sind:

- parteiliche Unterstützung

- Selbstbestimmung fördern

- keine Diskriminierung dulden

- Selbstachtung und Achtung anderer entwickeln

- Vertrauen schaffen durch Vertrautsein

- Kenntnis der eigenen Stärken und Schwächen vertiefen

- Das Interesse am »Peer", also am Gleichbetroffenen, zeigen

- Emanzipation von traditionellen Rollenerwartungen

Der angesprochene Innovationsdruck betrifft vor allen Dingen die Vertragsstaaten: Sie müssen die Voraussetzung schaffen, damit die UN-Behindertenrechtskonvention Wirklichkeit werden kann. Es ist also zunächst öffentliches Recht angesprochen. Und dieses hat

\section{"Mit einem Index kann die Inklusions-}

\section{tauglichkeit einer Stadt berechnet werden«}

Bemerkenswert ist auch der Artikel 26 (Habilitation und Rehabilitation). Dort wird die Unterstützung behinderter Menschen durch behinderte Menschen angesprochen. Die UN-Behindertenrechtskonvention setzt also auch auf die Selbstorganisation behinderter Menschen, auf Menschenkraftentwicklung. Wir kennen in diesem Zusammenhang den Begriff "Habilitation « kaum. Im »Sozialchinesisch" spielt er keine Rolle. Deshalb empfiehlt es sich, ins lateinische Lexikon zu schauen, um den inhaltlichen Schwerpunkt von Habilitation besser begreifen zu können. Habilitare wird wie folgt übersetzt: an sich, in sich haben, zeigen, mit sich bringen, verursachen, vermögen, können. Hier wird das angesprochen, was in der Peer-support-Bewegung als »Menschenrechtsorientierung " gemeint ist.

"Peer support « wird vor allen Dingen im amerikanischen Erfahrungsfeld als eine Methode des "Peer Counseling « bezeichnet. Behinderte beraten und unterstützen Behinderte. Die Prinzipien von »Peer support" und »Peer coun- mittelbar Konsequenzen auf alle Akteure des Sozialstaates. Es geht um eine inklusive Gestaltung des gesamten öffentlichen Lebens, sofern die staatlichen Stellen (Bund, Länder, Gemeinden, Sozialversicherungsträger, öffentliche Kostenträger etc.) Einfluss nehmen können über die spezifischen Gesetzgebungs- und Verwaltungskompetenzen. Es geht um einen Paradigmenwechsel, der einzuordnen ist in eine allgemeine sozialökologische Entwicklung.

\section{Sozialökologische Entwicklung}

Was ist mit sozialökologischer Entwicklung gemeint? In den westlichen Gesellschaften geht es im Prinzip um die Thematisierung des Humanen in einem strukturellen Konflikt. Einerseits gibt es die bekannten Strategien der »Fabrikation des zuverlässigen Menschen« für den Markt. Andererseits gibt es die Versuche der Selbstbestimmung des Menschen als Organisator seines eigenen Lebensglücks. Und das Individuum ist hin- und hergezogen in diesem Spannungsfeld. Wenn man es pointiert ausdrücken will: Die UNBehindertenrechtskonvention steht auf der Seite des individuellen Lebensglücks für jeden Menschen, also auch für behinderte Menschen. In diesem sozialökologischen Kontext gehören:

- Die Aufforderung zur Entwicklung eines anderen Wachstumsmodells, das auch Gesichtspunkte des Lebensglücks beachtet. (3)

- Der wachsende weltweite Partizipationswille der Bürgerinnen und Bürger, der oft als » Graswurzelrevolution « bezeichnet wird.

- Der Einfluss der amerikanischen Frauenbewegung auf die Gestaltung der Sozialen Arbeit. Es geht darum, dass wir pflegebedürftige Menschen nicht wie Gegenstände behandeln dürfen. Wir sind aufgefordert, achtsam mit ihren Bedürfnissen umzugehen und sie menschenfreundlich $\mathrm{zu}$ behandeln. (4)

- Die Durchsetzung des Prinzips der Sozialraumorientierung in der Sozialarbeit. Sozialraumorientierung wird doppelt bezogen definiert: Einerseits geht es um die Ressourcen, die im privaten Bereich liegen und die zur Lösung von Problemen aktiviert werden können. Andererseits geht es um eine öffentliche Orientierung. Zur Lösung von sozialen Problemen sollen möglichst die Ressourcen und Angebote genutzt werden, die im unmittelbaren Lebensraum der Hilfesuchenden zu aktivieren sind. (5)

- Der Versuch eine »menschenfreundliche « Architektur zu gestalten. Hier gibt es seit Jahren Überlegungen im Sinne einer Mustersprache, die uns Mut machen will zur menschenfreundlichen Umweltgestaltung. (6)

Der zentrale Treiber all dieser Aktivitäten ist aber im Sinne der UN-Behindertenrechtskonvention die Kraft der Menschenrechtsentwicklung.

\section{Dritter Sozialraum}

Das ist ein Begriff, der von dem Psychiater Klaus Dörner geprägt wurde. Er ist ein Vertreter und Verfechter der »Entinstitutionalisierung und der Heimauflösung«. Er bezeichnete im Jahr 2002 »Heimträger als Geiselnehmer«. Das dürfte für viele Heimangebo- 
te eine zutreffende Beschreibung sein. Ich selbst kenne die Praxis der »Geiselnahme" aus der Wohnungslosenhilfe, als ich für mehrere Jahre Leiter eines Obdachlosenasyls war. Wir hatten damals festgestellt, dass grundsätzlich alle alleinstehenden Wohnungslosen der Stadt in diesem Asyl aufgenommen wurden. Man hat sich nicht die Mühe einer sorgfältigen Diagnose gemacht; denn so hätte man feststellen können, dass die überwiegende Anzahl der im Heim aufgenommenen durchaus noch in der Lage waren, normal zu wohnen - mit oder ohne Betreuung.

Dörner beschreibt den dritten Sozialraum als den zivilgesellschaftlichen Raum. Der erste Sozialraum ist der private; der zweite Sozialraum ist der staatliche Sozialraum. Eine wirkliche Inklusion sei nur in der Nachbarschaft möglich, insofern setzt er auf die Entwicklung zivilgesellschaftlicher Dynamik. Dörner setzt auf örtliche Lösungen.

\section{Strategische Weichenstellung}

Wenn man das Stichwort von Klaus Dörner aufnehmen will - »Heimträger als Geiselnehmer" - dann ist man mitten drin in einem zentralen Bereich der Sozialwirtschaft. Was ist damit gemeint?

Inklusion vor Ort kann nur über eine örtliche Teilhabeplanung organisiert werden. (7) Diese kann nur dann gelingen, wenn sie begleitet wird durch die Beteiligung behinderter Bürgerinnen und Bürger. Diese werden eine lebensraumzentrierte Unterstützung fordern. Hier dürfte es regelmäßig eine entsprechende Nachfrage und entsprechende Bedarfe geben. Nun werden vorhandene Leistungsträger im Bereich der Sozialwirtschaft oft eine lebensraumferne Unterstützung anbieten wollen, weil ihre Angebote einer besonderen Strategie folgen. Hier geht es um induzierte Nachfragen und Bedarfe. Bei der Erarbeitung einer örtlichen Teilhabeplanung ist also darauf zu achten, dass über eine strategische Weichenstellung die Voraussetzungen geschaffen werden können in Richtung auf vermehrte inklusive Angebote in einem entsprechend zusammengestellten Wohlfahrtsmix.

In der Behindertenhilfe geht es um eine gewaltige Summe. Gegenwärtig werden rund zwölf Milliarden Euro im
Jahr ausgegeben. Die Frage muss gestellt werden, ob man mit diesem vielen Geld intelligenter umgehen kann, wenn die Prinzipien beachtet werden, die über die UN-Behindertenrechtskonvention ins Spiel gekommen sind.

Wenn es bei der Kontextgestaltung gut läuft, dann sind wir bei einem Verständnis von der Gestaltung konkreter Lebensbedingungen im Sinne eines »Design für alle « angekommen: Was für behinderte Menschen gut ist, ist auch für alte Menschen gut; und was für beide Gruppen gut ist, das ist dann für uns alle gut. Das ist ein Satz, der aus der Konzeption des »Designs für alle« stammt.

\section{Vision}

Die entscheidende Vision wird in der UN-Behinderten rechtskonvention selbst formuliert. Aus der Perspektive der Menschenrechtsentwicklung geht es zusammenfassend um

- die Achtung der Menschenwürde, unabhängig davon, ob ein Mensch behindert ist oder nicht. Hier geht es im theologischen Sinne um die Gestaltung der Hilfe im Sinne einer Gottesebenbildlichkeit;

- die Beteiligung der Betroffenen an der Gestaltung der Lebensbezüge;

- die Teilhabe an allen gesellschaftlichen Möglichkeiten nach der Maßgabe der individuellen Potenziale;

- die Gestaltung örtlicher Wirklichkeit in der Weise, dass dort ein inklusives Leben für jeden Menschen gelingen kann;

- den Abbau von Diskriminierung und Ausschluss;

- die Möglichkeit der Rechtsverwirklichung für jeden unabhängig von dessen finanzieller Kraft;

- die Entwicklung eines öffentlichen Bewusstseins über Bedingungen und Konsequenzen von Inklusion.

Als Modell wird eine »inklusive Gemeinde " beschrieben. Dies hat die "Montagstiftung Jugend und Gesellschaft « getan, indem sie ein Praxishandbuch veröffentlicht hat zum Thema »Inklusion vor Ort«. (8)

\section{Inklusion vor Ort}

In dem Praxishandbuch gibt es ein Verfahren, mit Hilfe dessen ein kommunaler Index berechnet werden kann, der dazu dient, die "Inklusionstauglichkeit « einer Stadt und ihrer Organisation festzustellen.

Wenn man sich dieses Verfahren genau anschaut, dann wird man feststellen, »dass der Teufel im Detail steckt«. Man begibt sich auf einen Prozess, der sich über Jahre entwickeln wird. Dieser Prozess muss aber nachhaltig verfolgt werden. Wir stehen erst am Anfang. Das ist wohl so, wie uns ein chinesisches Sprichwort sagt: Auch der längste Weg beginnt mit einem ersten Schritt.

Der kommunale Index bietet ein System von Fragen, mit denen sich Betroffene und Beteiligte vor Ort auseinandersetzen können, um die inklusive Qualität eines Gemeinwesens beurteilen zu können. Die entsprechenden Fragen können nicht immer mit Ja oder Nein beantwortet werden, die Beantwortung dieser Fragen soll vor allem eine Auseinandersetzung um inklusive Standards auslösen.

Wir müssen uns nun nicht alle Fragen anschauen; deutlich soll das Prinzip werden. Deshalb hier die Fragen zu den Gesichtspunkten von »Wohnen und Versorgung «:

1. Gibt es speziell für junge Menschen Zugang zu öffentlichen Plätzen und Räumen, die sie als Treffpunkte und zum selbstbestimmten Zeitvertreib nutzen können?

2. Gibt es Unterstützungsangebote für Menschen, die wichtige Dinge des täglichen Bedarfs nicht eigenständig erledigen können?

3. Gibt es überall Zugang zur ärztlichen Versorgung?

4. Gibt es mobile Versorgungsangebote in ländlichen, dünn besiedelten Gebieten?

5. Gibt es ausreichend Zugang zu öffentlichen Parks, Grünflächen und Sportflächen etc.?

6. Ist es für alle selbstverständlich, Wohnungslose als gleichberechtigte Mitglieder der Gemeinschaft anzuerkennen?

7. Sind überall Geschäfte für die tägliche Versorgung schnell erreichbar (Lebensmittel, Apotheken, Banken, Kleidung)?

8. Gibt es öffentlich zugängliche Toilet- 
ten, die für alle gut erreichbar, sicher und in einem ordentlichen Zustand sind?

9. Gibt es Unterstützungsangebote für Menschen mit spezifischem Bedarf adäquaten und bezahlbaren Wohnraum zu finden?

10. Gibt es für alle Menschen adäquaten und bezahlbaren Wohnraum?

Wir sehen hier, dass das Prinzip des »Designs für alle« schon Wirkung zeigt. Wenn diese Prinzipien beachtet werden, dann entsteht langsam auch für behinderte Menschen eine inklusive Wirklichkeit.

Ein abschließendes Wort: Das Ganze ist ein gewaltiges Projekt. Vielleicht sagen dann einige, dann fangen wir doch gar nicht erst an; der gegenwärtige Zustand ist doch in Ordnung. Dörner warnt vor solchen Fragestellungen und dem damit zusammenhängenden Gewöhnungseffekt, der durch die eingefahrene Praxis entstanden ist. Jeder und jede möge sich aufrichtig befragen: »Willst Du wirklich so leben, wie $\mathrm{Du}$ das gegenwärtig dem behinderten Menschen in dem Kontext, den Du zu verantworten hast, zumutest?"

Inklusion beginnt mit Selbstreflexion und führt über die Sozialrechtsverwirklichung in die Menschenrechtsverwirklichung. Hier müssen wir offensichtlich einen Schwerpunkt setzen.

\section{Anmerkungen}

(1) Die Stellungnahme ist im Internet zu finden: www.vsop.de.

(2) Die Artikel sind zitiert nach: Übereinkommen der Vereinten Nationen über die Rechte von Menschen mit Behinderungen, BMAS, Bonn 2011.

(3) Vgl. dazu: Petra Pinzler, Immer mehr ist nicht genug, München 2011.

(4) Vgl. dazu: Elisabeth Conradi, Take Care, Frankfurt am Main 2001.

(5) Vgl. dazu: Wolfgang Budde, Frank Früchtel, Sozialraumorientierte Soziale Arbeit, in: NDV 7 (2005), S. 238-242 und 8 (2005), S. 287292.

(6) Vgl. dazu: Christopher Alexander u. a., Eine Mustersprache, Wien 1995.

(7) Vgl. dazu: Doris Rüter, Örtliche Teilhabeplanung am Beispiel der Stadt Münster, Münster 2011.

(8) Vgl. dazu: Inklusion vor Ort, DVEigenverlag Berlin 2011.

\section{"Standardwerk für Berater im Sozialrecht"}

RAin Birgit Scheibe, Sozialrecht aktuell 3/10

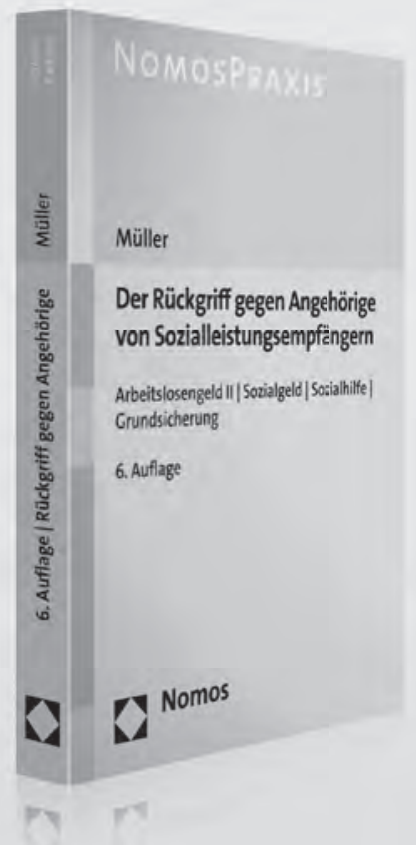

Aus dem Inhalt:

- die Grundzüge des reformierten Unterhaltsrechts

- Möglichkeiten und Grenzen der Inanspruchnahme naher Angehöriger beim Bezug von Sozialhilfe

- Regress beim Bezug von Arbeitslosengeld II und Sozialgeld nach SGB II

- Einfluss von Unterhaltsansprüchen.

Die Neuauflage untersucht nicht nur die Rückgriffsmöglichkeiten beim Sozialhilfebezug (einschließlich der Grundsicherungsleistungen), sondern auch die unterhaltsrechtlichen Auswirkungen im Rahmen des SGB II. Die Werte der aktuellen Düsseldorfer Tabelle sowie die aktuelle Rechtsprechung im Unterhaltsrecht sind berücksichtigt. Die unterhaltsrechtlichen Leitlinien des OLG Celle bilden die Grundlage der zahlreichen Berechnungsbeispiele. "Das Werk verdient in jeder Hinsicht eine besondere Empfehlung."

Christian Fritz, FASozR, Betreuungsmanagement 2/09, zur Vorauflage

Weitere Informationen: www.nomos-shop.de/14635

\section{Der Rückgriff gegen}

Angehörige von Sozialleistungsempfängern

Arbeitslosengeld II, Sozialgeld, Sozialhilfe, Grundsicherung Von Prof. Dr. Christian Müller 6. Auflage 2012, 218 S., brosch., 39,-€

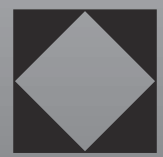
Nomos 